\title{
Nanoparticle Formation and Behavior in Turbulent Spray Flames Investigated by DNS
}

\author{
Abouelmagd Abdelsamie ${ }^{1,2}$ (D) Frank Einar Kruis ${ }^{3} \cdot$ Hartmut Wiggers $^{3,4}$. \\ Dominique Thévenin ${ }^{1}$
}

Received: 25 October 2019 / Accepted: 13 April 2020 / Published online: 22 May 2020

(c) The Author(s) 2020

\begin{abstract}
This work is a first direct numerical simulation of a configuration closely related to the SpraySyn burner (Schneider et al. in Rev Sci Instrum 90:085108, 2019). This burner has been recently developed at the University of Duisburg-Essen to investigate experimentally nanoparticle synthesis in spray flames for a variety of materials. The present simulations are performed for ethanol and titanium tetraisopropoxide as a solvent and precursor, respectively, in order to produce titanium dioxide nanoparticles. In the direct numerical simulations, the complete scenario leading to the production of well-defined nanoparticles is taken into account, including evaporation of the liquid mixture (solvent and precursor) injected as a spray, multi-step kinetics for gas-phase combustion, and finally nanoparticle synthesis. The employed models are described in this article. Additionally, the impact of the inlet velocity of the pilot flame on the nanoparticle synthesis is investigated. It has been found that increasing this speed delays spray flame ignition, decreases nanoparticle concentration, but leads to a narrower size distribution at early stage.
\end{abstract}

Keywords Direct numerical simulation $\cdot$ Spray flame $\cdot$ Nanoparticle PSD

\section{Introduction}

Nanoparticles are found in many environmental processes and in an increasing number of industrial applications. For example, they are observed as soot particles in combustion processes, as dust during volcanic eruptions, as color pigments in paints or in cosmetic

Abouelmagd Abdelsamie

abouelmagd.abdelsamie@ovgu.de

1 Laboratory of Fluid Dynamics and Technical Flows, University of Magdeburg "Otto von Guericke”, Universitätsplatz 2, 39106 Magdeburg, Germany

2 Lab of Fluid Mechanics, Faculty of Engineering (Elmattaria), Helwan University, Cairo 11311, Egypt

3 CENIDE, Center for Nanointegration Duisburg-Essen, University of Duisburg-Essen, 47057 Duisburg, Germany

4 IVG, Institute for Combustion and Gas Dynamics - Reactive Fluids, University of Duisburg-Essen, Duisburg, Germany 
products. They are also used for drug delivery and cancer therapy. Further examples are carbon black in tires, metallic nanoparticles as catalysts in chemical reactors, etc.

During the last decade many studies have been devoted to nanoparticle synthesis from gaseous flames. Now, this process appears to be relatively well established (Kammler et al. 2001; Janzen et al. 2003; Roth 2007; Li et al. 2016; Mädler et al. 2002). However, the focus is currently set on nanoparticle synthesis from spray flames (Mädler et al. 2002; Mueller et al. 2003; Weise et al. 2015; Rittler et al. 2017). This is because the conventional gasphase processes (synthesis from a gaseous flame) require precursors that are either gaseous or that can be vaporized and mixed with the burner gases before they react within the reaction chamber. Unfortunately, such precursors are only available for a limited number of elements and are often based on metal chlorides, metal organics or organometallics that are very expensive and/or toxic (Schneider et al. 2019). Recently, a collaborative research initiative was started in Germany (SPP1980 funded by the German Research Foundation-DFG-entitled "Nanoparticle Synthesis in Spray Flames SpraySyn: Measurement, Simulation, Processes") to create a reference configuration, the "SpraySyn-burner" to investigate in a systematic manner nanoparticle formation in spray flames. This burner shall deliver benchmark data for the corresponding research community (Schneider et al. 2019). One of the advantages of this burner is that it is designed from the start while taking into account the bottlenecks of companion numerical simulations; for example, the gas feed for the pilot flame in this burner is injected through a thick porous area (flat flame), facilitating simulations since a very fine grid is not needed there.

For the SpraySyn burner, most existing simulations rely on Large Eddy Simulations, like for instance those documented in Rittler et al. (2017) and Schneider et al. (2019). The present authors are involved as well in this collaborative DFG project, with the ultimate objective of providing DNS results for conditions as close as possible to those found in the real SpraySyn burner. To the authors' knowledge, this publication is a first DNS of a configuration close to the full SpraySyn burner. The ability of DNS to capture the complete process is evaluated by taking into account step-by-step the different processes controlling nanoparticle production from a spray flame. The liquid mixture injected as a spray consists of ethanol (solvent) and titanium tetraisopropoxide (precursor); it is used to produce titanium dioxide $\left(\mathrm{TiO}_{2}\right)$ nanoparticles. The impact of the inlet velocity of the pilot flame on the particle production will be investigated as well.

This paper is organized as follows: in Sect. 2, all numerical models are described; the geometrical configuration is presented in Sect. 3, followed by a discussion of the numerical results in Sect. 4, before concluding in Sect. 5.

\section{Numerical Approaches}

In this study three main numerical approaches are employed: (1) DNS to solve for the gas phase and the corresponding chemical reactions; (2) a Lagrangian description for tracking spray droplets, and (3) an Eulerian approach to model the processes controlling the evolution of the nanoparticles (growth, aggregation, coagulation...). Droplets, being noticeably smaller than the grid resolution, are modeled as point droplets with a variable diameter. All numerical models are integrated into the in-house DNS code called DINO, a Fortran90 code developed by our group during the last 10 years. A 6th-order central finite-difference approach is used for the spatial discretization, while a semi-implicit 3rd-order Runge-Kutta method is employed for temporal integration. The open-source library Cantera 2.4.0 (Goodwin et al. 2015) is used 
to compute all chemical reactions, thermodynamic terms, and molecular transport processes in the gas phase. More details about DINO can be found in particular in Abdelsamie et al. (2016) and Chi et al. (2017, 2018, 2020). In this code, the low Mach number formulation is implemented in order to get a highly efficient DNS solver, while adding source terms (written as $\Gamma$ ) representing the coupling with the disperse phase (e.g., spray evaporation). The conservation equations for an ideal gas involving $N_{s}$ chemical components are implemented as follows:

$$
\begin{gathered}
\frac{\partial \rho}{\partial t}+\frac{\partial\left(\rho u_{i}\right)}{\partial x_{i}}=\Gamma_{m} \\
\frac{\partial\left(\rho u_{i}\right)}{\partial t}+\frac{\partial\left(\rho u_{j} u_{i}\right)}{\partial x_{j}}=-\frac{\partial \tilde{p}}{\partial x_{i}}+\frac{\partial \tau_{i j}}{\partial x_{j}}+\Gamma_{u i} \quad(i=1,2,3), \\
\frac{\partial T}{\partial t}+u_{j} \frac{\partial T}{\partial x_{j}}=\frac{1}{\rho C_{p}}\left[\frac{\partial}{\partial x_{j}}\left(\lambda \frac{\partial T}{\partial x_{j}}\right)-\frac{\partial T}{\partial x_{j}} \sum_{k=1}^{N_{s}} \rho C_{p, k} Y_{k} \theta_{k, j}-\sum_{k=1}^{N_{s}} h_{k} \dot{\omega}_{k}+\Gamma_{T}\right], \\
\rho \frac{\partial Y_{k}}{\partial t}+\rho u_{j} \frac{\partial Y_{k}}{\partial x_{j}}=-\frac{\partial\left(\rho Y_{k} \theta_{k j}\right)}{\partial x_{j}}+\dot{\omega}_{k}+\Gamma_{m, k},
\end{gathered}
$$

This system of equations is closed by (1) the ideal gas law, and (2) the additional condition describing overall mass conservation:

$$
\begin{gathered}
\rho=\frac{P W}{\mathrm{R} T}, \\
\sum_{k=1}^{N_{s}} Y_{k}=1 .
\end{gathered}
$$

In these equations, $\rho, u_{i}, \tilde{p}, P, T, Y_{k}, N_{s}, \mathrm{R}$ and $W$ are the density of the gas mixture, $i$-thcomponent of flow velocity, fluctuation pressure, thermodynamic pressure, gas temperature, $k$-th species mass fraction, number of species, ideal gas constant, and mixture mean molecular weight, respectively. In Eq. (2) $\tau_{i j}$ is the viscous stress tensor,

$$
\tau_{i j}=\mu\left(\left[\frac{\partial u_{i}}{\partial x_{j}}+\frac{\partial u_{j}}{\partial x_{i}}\right]-\frac{2}{3} \delta_{i j}\left[\frac{\partial u_{l}}{\partial x_{l}}\right]\right),
$$

where $\delta_{i j}$ and $\mu$ are the Kronecker delta, and dynamic viscosity, respectively. In Eqs. (3) and (4), $C_{p}, h_{k}, \dot{\omega}_{k}, \lambda$, and $\theta_{k, j}$ represent the specific heat capacity at constant pressure, specific enthalpy, mass reaction rate, heat diffusion coefficient and $j$-th component of the species molecular diffusion velocity, respectively. Additionally, $\Gamma_{m}, \Gamma_{u_{i}}$, and $\Gamma_{T}$ are the liquid source terms for mass, momentum, and temperature equations, respectively,

$$
\Gamma_{m}=-\frac{1}{\Omega} \sum_{d} \alpha_{d} \frac{d m_{d}}{d t},
$$




$$
\begin{gathered}
\Gamma_{u_{i}}=-\frac{1}{\Omega} \sum_{d} \alpha_{d} \frac{d\left(m_{d} V_{i, d}\right)}{d t}, \\
\Gamma_{T}=-\frac{1}{\Omega} \sum_{d} \alpha_{d} \frac{d\left(m_{d} C_{p, L} T_{d}\right)}{d t} .
\end{gathered}
$$

Concerning the species transport equations, the source term is

$$
\Gamma_{m, k}=\Gamma_{m} \delta_{k, f},
$$

where $m_{d}=\rho_{L} \pi a_{d}^{3} / 6$ is the mass of the liquid droplets with diameter $a_{d}$ and density $\rho_{L}$; $V_{i, d}$ is $i$-th component of a droplet $d, T_{d}$ is the temperature of a droplet $d, C_{p, L}$ is the specific heat of a liquid droplet $d$ at constant pressure, and $\delta_{k, f}$ is 1 for the fuel species, 0 otherwise. The quantities $\alpha_{d}$ and $\Omega$ are the interpolation weight and the volume of the Cartesian grid cell centered on the DNS node, respectively. In the code three different molecular diffusion models are available, with increasing level of accuracy, complexity, and computational cost: (1) unity Lewis numbers; (2) mixture-averaged diffusion velocities; (3) multicomponent diffusion velocities. In the present simulations, the intermediate approach (mixtureaveraged approach) has been activated for all diffusion terms in DINO, as good compromise between accuracy and complexity.

A two-way coupling between gas and liquid phase is implemented via the exchange of mass, momentum and energy. The droplet equations rely on the model first introduced by Abramzon and Sirignano (1989), taking into account the improvements suggested in Kitano et al. (2014). The implemented equations describing droplet location, momentum, mass transfer, and heat transfer read as follows (Abdelsamie and Thévenin 2017, 2019):

$$
\begin{gathered}
\frac{\mathrm{d} \mathbf{X}_{d}}{\mathrm{~d} t}=\mathbf{V}_{d}, \\
\frac{\mathrm{d} \mathbf{V}_{d}}{\mathrm{~d} t}=\frac{\mathbf{U}_{\infty}-\mathbf{V}_{d}}{\tau_{v, d}}, \\
\frac{\mathrm{d} a_{d}^{2}}{\mathrm{~d} t}=-\frac{a_{d}^{2}}{\tau_{a, d}}, \\
\frac{\mathrm{d} T_{d}}{\mathrm{~d} t}=\frac{1}{\tau_{T, d}}\left[T_{\infty}-T_{d}-\frac{\mathrm{B}_{T, d} L_{v}}{C_{p, f}^{F} W_{F}}\right] .
\end{gathered}
$$

In Eqs. (12)-(15), there are four different subscripts and superscripts: $\infty, F, f$, and $L$, which are standing for variables in the far-field gaseous region, properties of fuel vapor in film region, mixture variable in film region, and liquid properties, respectively. The quantities $\mathbf{V}_{d}$ and $\mathbf{U}_{\infty}$ are the velocity of the $d$-th droplet and of the surrounding gas at droplet location $\mathbf{X}_{d}$. Also, $T_{\infty}, L_{v}, W_{F}, C_{p, f}^{F}$ and $\mathrm{B}_{T, d}$ are mixture temperature in far-field, molar latent heat of droplet vaporization, molar mass of the fuel, specific heat of the fuel vapor in the film region and heat transfer number, respectively. The properties and variables in the film region are computed based on the one-third rule (Abramzon and Sirignano 1989; Wang and Rutland 2007) and have the subscript $f$, as mentioned above. Motion and evaporation 
of the droplets are characterized by three characteristic time scales: momentum relaxation time $\left(\tau_{v, d}\right)$, evaporation delay $\left(\tau_{a, d}\right)$ and heating delay $\left(\tau_{T, d}\right)$, given by:

$$
\begin{gathered}
\tau_{v, d}=\frac{\rho_{L} a_{d}^{2}}{18 \mu_{f}\left(1+\frac{1}{6} \mathrm{Re}_{d}^{2 / 3}\right)}, \\
\tau_{a, d}=\frac{\mathrm{Sc}_{d}}{4 \mathrm{Sh}_{d}} \frac{\rho_{L}}{\mu_{f}} \frac{a_{d}^{2}}{\ln \left(1+\mathrm{B}_{m, d}\right)}, \\
\tau_{T, d}=\frac{\operatorname{Pr}_{d}}{6 \mathrm{Nu}_{d}} \frac{C_{p, L}}{C_{p, f}} \frac{\rho_{L} a_{d}^{2}}{\mu_{f}} \frac{\mathrm{B}_{T, d}}{\ln \left(1+\mathrm{B}_{T, d}\right)} .
\end{gathered}
$$

In these equations, the characteristic time scales are computed as a function of various dimensionless numbers: the droplet Reynolds number, $\mathrm{Re}_{d}$, the Spalding mass transfer number $\left(\mathrm{B}_{m}\right)$ and the heat transfer number $\left(\mathrm{B}_{T}\right)$,

$$
\begin{gathered}
\mathrm{Re}_{d}=\frac{\rho_{\infty} a_{d}\left|\mathbf{U}_{\infty}-\mathbf{V}_{d}\right|}{\mu_{f}}, \\
\mathrm{~B}_{m, d}=\frac{Y_{s, d}-Y_{F, \infty}}{1-Y_{s, d}}, \\
Y_{s, d}=\frac{W_{F}}{W_{F}+W_{O}\left(P_{\infty} / P_{\mathrm{sat}, d}-1\right)}, \\
\mathrm{B}_{T, d}=\left(1+\mathrm{B}_{m, d}\right)^{\phi}-1, \\
\phi=\frac{C_{p, f}^{F}}{C_{p, f}} \frac{\mathrm{Sh}_{d}}{\mathrm{Nu}_{d}} \frac{\operatorname{Pr}_{d}}{\mathrm{Sc}_{d}} .
\end{gathered}
$$

Here, $Y_{s, d}, Y_{F, \infty}, W_{O}, P_{\infty}$ and $P_{\text {sat }, d}$ are the saturated vapor mass fraction, fuel mass fraction in far-field gas mixture, oxidizer molar mass, far-field pressure and saturated vapor pressure computed with the Clausius-Clapeyron equation:

$$
P_{\text {sat }, d}=P_{\text {ref }} \exp \left[-\frac{L_{v}}{R}\left(\frac{1}{T_{d}}-\frac{1}{T_{\text {ref }}}\right)\right] .
$$

In Eq. (24), $P_{\text {ref }}$, and $T_{\text {ref }}$ are reference pressure and temperature, taken here as atmospheric pressure and boiling temperature of the fuel at this pressure, respectively, while $L_{v}$ is corrected using the Watson equation,

$$
L_{v}=L_{v, s}\left(\frac{T_{\mathrm{cr}}-T_{d}}{T_{\mathrm{cr}}-T_{\mathrm{ref}}}\right)^{0.38} .
$$


Here, $L_{v, s}$ and $T_{\mathrm{cr}}$ are the molar latent heat at reference temperature $T_{\text {ref }}$, and critical temperature of the fuel, respectively. As shown in Eqs. (22)-(23) the heat transfer number depends on the fuel vapor to gas mixture specific heats $\left(C_{p, f}^{F}, C_{p, f}\right)$ at film region, Prandtl number (Pr), Schmidt number (Sc), Sherwood number (Sh), and Nusselt number (Nu), which are computed following Ref. Borghesi et al. (2013):

$$
\begin{gathered}
\mathrm{Nu}_{d}=2+\frac{\mathrm{Nu}_{d, 0}-2}{F\left(\mathrm{~B}_{T, d}\right)}, \\
\mathrm{Sh}_{d}=2+\frac{\mathrm{Sh}_{d, 0}-2}{F\left(\mathrm{~B}_{m, d}\right)}, \\
\mathrm{Nu}_{d, 0}=1+\left(1+\operatorname{Re}_{d} \mathrm{Sc}_{d}\right)^{1 / 3} f\left(\operatorname{Re}_{d}\right), \\
\mathrm{Sh}_{0}=1+\left(1+\operatorname{Re}_{d} \operatorname{Pr}_{d}\right)^{1 / 3} f\left(\operatorname{Re}_{d}\right), \\
F\left(\mathrm{~B}_{d}\right)=\left(1+\mathrm{B}_{d}\right)^{0.7} \ln \left(1+\mathrm{B}_{d}\right) / \mathrm{B}_{d}, \\
f\left(\operatorname{Re}_{d}\right)=\max \left(1 ; \operatorname{Re}_{d}^{0.077}\right) .
\end{gathered}
$$

This coupling between the gas phase and the liquid phase is a combination of DNS (for the gas phase) and discrete particle simulation technique (DPS) for the disperse phase, and can thus overall be written DNS-DPS.

Concerning now the third modelling level, used to describe the evolution of the nanoparticles, the model developed by Kruis et al. (1993) with improvements described in Panda and Pratsinis (1995) and Weise et al. (2015) has been implemented. This model can be summarized as follows.

$$
\begin{gathered}
\frac{d N}{d t}=-\frac{1}{2} \beta N^{2}+I, \\
\frac{d A}{d t}=-\frac{1}{\tau_{s}}\left(A-N a_{s}\right)+I a_{0}, \\
\frac{d \mathcal{V}}{d t}=I v_{0} .
\end{gathered}
$$

In these equations, $N$ is the nanoparticles' concentration, $A$ is the total surface area concentration, $\mathcal{V}$ is the total volume concentration, $v_{0}$ is the monomer volume, $a_{0}$ is the monomer surface area, and $I$ is the nucleation rate. These equations are discretized using a sixth-order, central finite-difference stencil identical to that used for the gas-phase equations (Abdelsamie et al. 2016). Additionally, an eighth-order filter (Kennedy and Carpenter 1994) has been activated for the nanoparticle equations, in order to eliminate spurious oscillations caused by the low diffusivity of the nanoparticles, which cannot be eliminated by the high-order central stencil employed for discretization. The coagulation kernel, $\beta$ is computed as 


$$
\begin{gathered}
\beta=4 \pi d_{c} \mathcal{D}\left[\frac{0.5 d_{c}}{d_{c}+g \sqrt{2}}+\frac{\mathcal{D} \sqrt{2}}{0.5 c d_{c}}\right]^{-1}, \\
g=\frac{\left[\left(d_{c}+L\right)^{3}-\left(d_{c}^{2}+L^{2}\right)^{1.5}\right]}{3 L d_{c}}-d_{c} \\
L=\frac{8 \mathcal{D}}{\pi c} \\
c=\sqrt{\frac{8 k_{b} T}{\pi \rho_{n} V}} \\
\mathcal{D}=\frac{k_{b} T}{3 \pi \mu d_{c}} .
\end{gathered}
$$

where, $L$ is the mean free path, $k_{b}$ is the Boltzmann constant, $\rho_{n}$ is the particle density, $T$ is the gas temperature, $\mu$ is the viscosity of the gas, $c$ is the particle velocity, and $\mathcal{D}$ is the diffusion coefficient of the particles. The primary diameter $d_{p}$, aggregate diameter $d_{a}$, and collision diameter $d_{c}$ are computed as follows

$$
\begin{gathered}
d_{p}=\left(\frac{6 \mathcal{V}}{A}\right), \\
d_{a}=\left(\frac{6 \mathcal{V}}{\pi N}\right)^{1 / 3}, \\
d_{c}=d_{p} n_{p}^{1 / 1.8}, \\
n_{p}=\frac{6 \mathcal{V}}{\pi N d_{p}^{3}} .
\end{gathered}
$$

In Eq. 33, the surface area of the completely fused particles

$$
a_{s}=\left(\frac{\mathcal{V}}{N v_{0}}\right)^{2 / 3} a_{0},
$$

and the characteristic sintering time $\tau_{s}$ for titanium dioxide are computed similarly to Buesser et al. (2011),

$$
\tau_{s}=7.44 \times 10^{16} T d_{p}^{4} \exp \left(\frac{31,000}{T}\right) .
$$




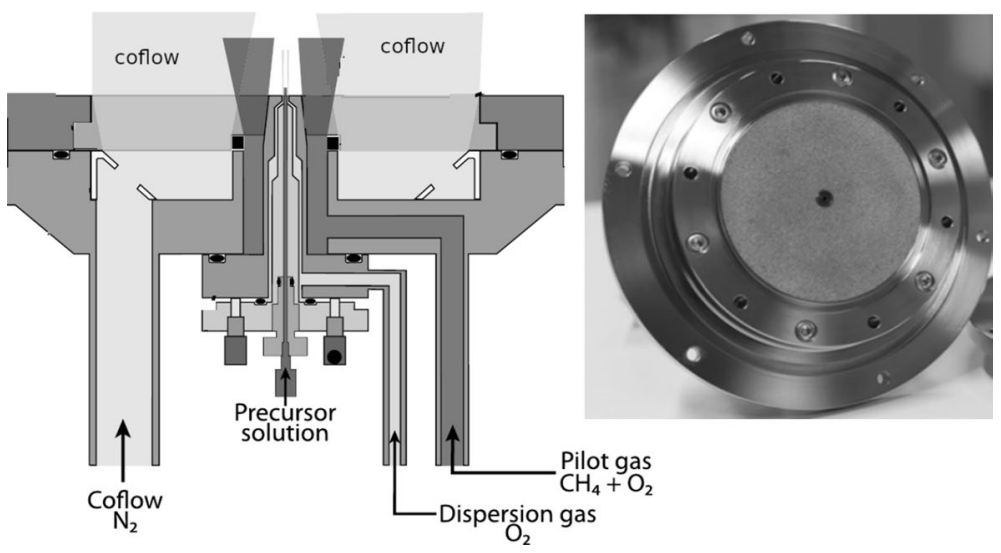

Fig. 1 Schematic diagram showing the real geometry of the SpraySyn burner (Schneider et al. 2019)

Table 1 Reaction mechanism employed to describe nanoparticle formation

\begin{tabular}{llc}
\hline Reaction & $A_{j}\left(\mathrm{~s}^{-1}\right)$ & $T_{a}(\mathrm{~K})$ \\
\hline $\mathrm{C}_{12} \mathrm{H}_{28} \mathrm{O}_{4} \mathrm{Ti}=2 \mathrm{H}_{2} \mathrm{O}+4 \mathrm{C}_{3} \mathrm{H}_{6}+\mathrm{TiO}_{2}$ & $3.96 \times 10^{5}$ & 8487 \\
\hline
\end{tabular}

\section{Numerical Configurations}

As it has been discussed before, the main purpose of this work is to have conditions representative of the SpraySyn burner in the DNS. In the experiments, the main solvent is ethanol, which is mixed (in liquid state) with a precursor and then injected together with a dispersion gas $\left(\mathrm{O}_{2}\right)$ through an injector. The liquid spray is then evaporated by the pilot flame $\left(\mathrm{CH}_{4} / \mathrm{O}_{2}\right)$. The layout of the burner can be seen in Fig. 1, where the liquid solution (ethanol+precursor) is injected through the central tube of the burner injector, while the pilot flame and the coflow enter through two annular regions surrounding the central injector. The complete description of the burner and experimental setup can be found in Ref. Schneider et al. (2019).

Kinetics play an important role for the final process outcome. In the current DNS simulation, a skeletal kinetic mechanism is used to describe ethanol oxidation. It consists of 35 species and 87 elementary reactions. This mechanism was developed and optimized at the University of Duisburg-Essen based on a large mechanism published in Marinov (1999). In the present study, a simple mechanism for titanium tetraisopropoxide (TTIP) has been used, as introduced in Ref. Weise et al. (2015). In this mechanism the conversion from TTIP to $\mathrm{TiO}_{2}$ is described by the single reaction given in Table 1 , where $A_{j}$ and $T_{a}$ are the preexponential constant and activation temperature in the Arrhenius law, respectively.

In the practical implementation, the first step is to compute the mass fraction of the precursor $y_{p}$ using the reaction from Table 1 ,

$$
\frac{d Y_{p}}{d t}=-A_{j} \exp \left(-\frac{T_{a}}{T}\right) Y_{p} .
$$

Then, the nucleation rate $I$ can be computed as follows: 


$$
I=-\frac{d Y_{p}}{d t} \frac{\rho N_{A}}{W_{p}} .
$$

The quantity $I$ constitutes the link between the gas and the solid phase, which is used to compute the mass reaction rate of the precursor in the gas phase, $\dot{\omega}_{p}$ :

$$
\dot{\omega}_{p}=-I Y_{p},
$$

where $N_{A}$ and $W_{p}$ are the Avogadro number and the molar mass of the precursor, respectively.

Obviously, DNS simulations of the large and complex configuration shown in Fig. 1 are extremely challenging. As a consequence, only the relevant part of the domain has been considered in this first DNS study, as illustrated in Fig. 2. The dimensions of this computational domain are $18 \mathrm{~mm} \times 9 \mathrm{~mm} \times 0.56 \mathrm{~mm}$ in streamwise, transverse, and crosswise directions, respectively. In the crosswise direction, only a small extension has been implemented, allowing the development of three-dimensional structures but reducing computational costs. The DNS domain is discretized over 16.7 million grid points with $17.6 \mu \mathrm{m}$ grid spacing. Moreover, the ratio between the laminar flame thickness of the pilot flame and the grid spacing is $\sigma_{f} / \Delta x=8.33$; therefore, the flame thickness is well resolved on the DNS grid. Additionally, several time scales should be resolved in these simulations: (1) the jet time scale $t_{j}=3.3 \times 10^{-6} \mathrm{~s}$, (2) the sintering time of the precursor $t_{p}=5 \times 10^{-7} \mathrm{~s}$, and (3) the ignition time scale $t_{g}=2.3 \times 10^{-4} \mathrm{~s}$. All of them have been properly resolved in the simulations, since the maximum (variable) time step is $\Delta t=3 \times 10^{-8} \mathrm{~s}$. Inflow/ outflow boundary conditions are used in the streamwise direction, while periodic boundary conditions are considered in the other directions. Inlet velocities similar to that found in the experiment have been considered: dispersion gas $\left(\mathrm{O}_{2}\right)$ is injected at a speed of $U_{j}=91.34 \mathrm{~m} / \mathrm{s}$, temperature of $T=500 \mathrm{~K}$, jet Reynolds number is 711 , and Mach number

Fig. 2 Schematic diagram showing the computational domain taken into account for the DNS simulations, projected onto the real burner (red rectangle)

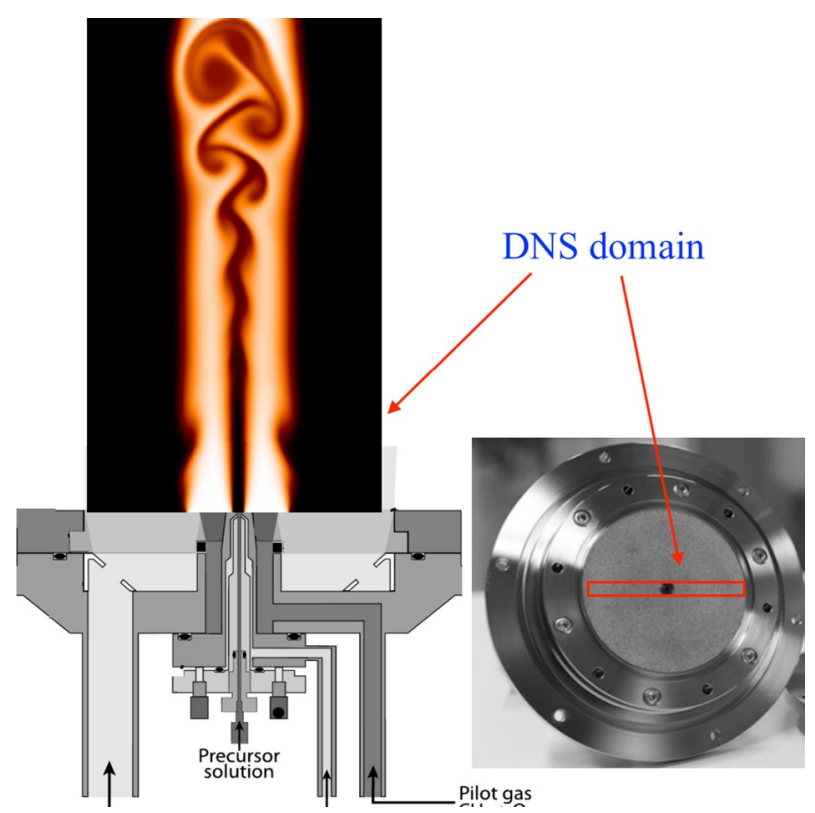


of 0.2 ; the pilot flame enters the domain at a speed of $U_{p}=3.71 \mathrm{~m} / \mathrm{s}$; finally, the coflow $\left(\mathrm{N}_{2}\right)$ is injected at a speed of $U_{c}=0.637 \mathrm{~m} / \mathrm{s}$ and temperature of $T=500 \mathrm{~K}$. The temperature of the pilot flame is determined based on the flame equivalence ratio, which is in the very lean regime; the resulting flame temperature is $T \approx 1570 \mathrm{~K}$. In the simulations, initially monodisperse liquid droplets (homogeneous liquid mixture of ethanol + TTIP), all starting with a diameter of $10 \mu \mathrm{m}$ and a temperature of $300 \mathrm{~K}$, are injected through a nozzle with a diameter of $d_{j}=0.3 \mathrm{~mm}$ at the same velocity as the dispersion gas. The pilot flame is injected through an annular area with inner and outer diameters of $1.2 \mathrm{~mm}$ and $3 \mathrm{~mm}$, respectively. The coflow starts being injected beyond the radius of $3 \mathrm{~mm}$. In order to trigger turbulence, fluctuation velocities with a turbulence intensity of $5 \%$ are added at the inlet boundary condition within the area of the central injection nozzle. This turbulent fluctuation field is first generated inside a separate box. An inverse fast Fourier transform (IFFT) is then used to generate a synthetic turbulence field (Abdelsamie et al. 2016). The generated field is stored in memory and injected plane after plane into the computational domain through the inflow on top of the mean flow.

\section{Results}

In this section the complete scenario of spray evaporation and nanoparticle generation will be discussed in details. Then, the impact of the inlet velocity of the pilot flame on nanoparticle synthesis will be investigated. Scatter plots and particle size distributions have been computed over the whole domain at different time instants. They will be discussed in this section to highlight the main chemicophysical processes dominating each stage. Since the present DNS simulations consider only a small part of the whole SpraySyn burner plenum, all effects that may occur further downstream cannot be captured. These DNS results depict the initial part of the process, starting with the injection of the very first spray droplets, leading later to spray flame ignition; the simulation is stopped when the remaining spray droplets start leaving the DNS domain through the top outflow. This limitation must be kept in mind when analyzing the present results.

\subsection{Spray evaporation and nanoparticle synthesis}

In order to describe nanoparticle formation from a spray flame, five figures will be presented at four different time instances $t=31 \tau_{j}, 46 \tau_{j}, 61 \tau_{j}$, and $74 \tau_{j}$, where $\tau_{j}=d_{j} / U_{j}$ is the jet time-scale, equal to $3.28 \mu$ s here. Figures 3 and 4 show a cut plane through the center of the numerical domain for the gas temperature in Fig. 3, and the ethanol mass fraction in the gas mixture in Fig. 4. Figures 5 and 6 present the scatter plots of the ethanol mixture fraction in the gas mixture versus gas temperature, and nanoparticle number concentration versus gas temperature, respectively. Finally, Figs. 7 and 8 show the histogram of primary and aggregate diameters of the nanoparticles, respectively.

\subsubsection{Evaporation}

When starting the experiments, the pilot flame is initiated into the domain to heat up the system. After the pilot flame burns in a stable manner, the spray starts being injected, as it can be observed from Fig. 3a. As soon as the droplets enter the domain, they are heated up and evaporation starts (Fig. 4a, b); as a consequence, the gas temperature 
Fig. 3 Spray flame evolution in time represented by a cut plane of gas temperature along the center of the domain. From left to right and from top to down the time is a $t=31 \tau_{j}, \mathbf{b} 46 \tau_{j}, \mathbf{c} 61 \tau_{j}$, d $74 \tau_{j}$, respectively. The black spheres mark the position of individual liquid droplets; the size of these spheres has been magnified for visualization purposes. Note that the color scale is changing for each subfigure, following the change in peak temperature
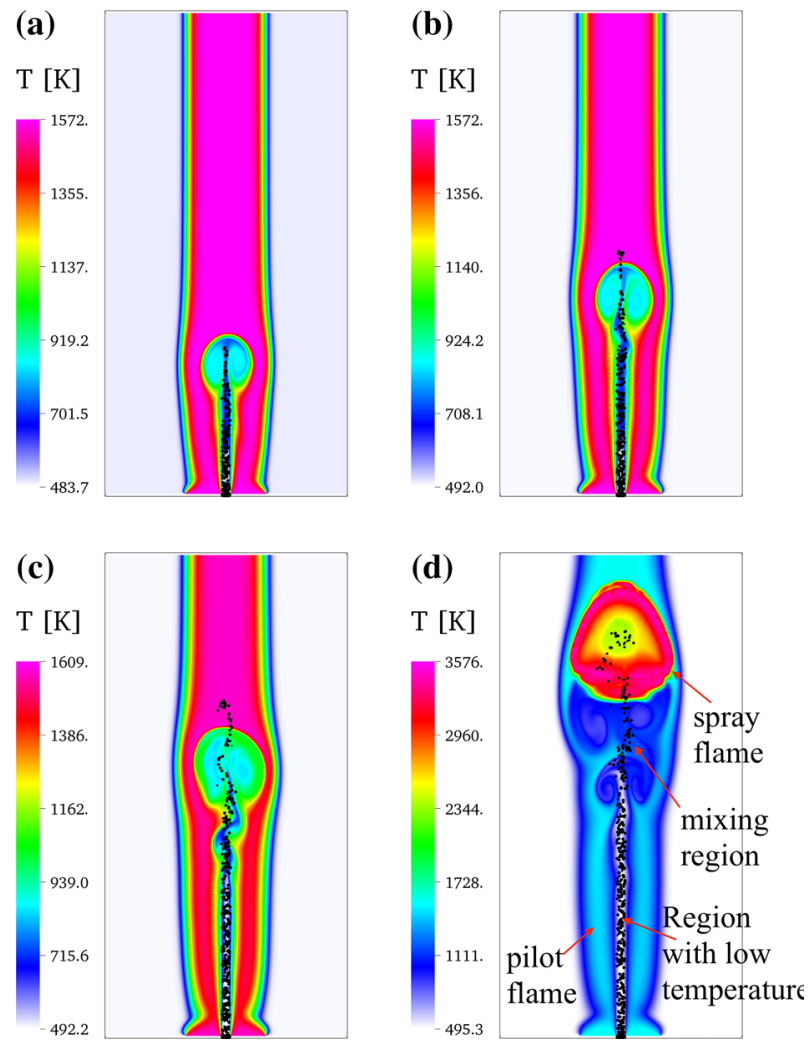

around the droplets is reduced, as it can be observed from Fig. $3 \mathrm{a}, \mathrm{b}$ at $t / \tau_{j}=31$ and 46. It is important to notice that the maximum temperature did not increase yet, since the spray did not ignite; at the same time, the ethanol concentration in the gas phase increases (Fig. 4a, b). Looking at the distribution of ethanol concentration in the gas mixture (Fig. 5a), it can be seen that ethanol evaporates first in a temperature range of $500 \leq T \leq 1300 \mathrm{~K}$, with maximum concentration found for $700<T<800 \mathrm{~K}$. At a later time (Fig. 3b), the spray starts escaping from the head of the dispersion jet head and enters the pilot flame region, at a much higher temperature. Therefore, the evaporation occurs now for at wider range of temperatures; ethanol concentration in the gas phase reaches its maximum value around peak temperature $T \approx 1570 \mathrm{~K}$ as it can be observed from Fig. $5 \mathrm{~b}$ at $t / \tau_{j}=46$.

At this still early stage of the evaporation process, the nanoparticle formation already starts (Fig. 6). Obviously, the nanoparticle number concentration is initiated at a very low value, as it can be observed from Fig. 6a (in which a zoom was necessary to make it visible at all). It rapidly increases to a significant amount (Fig. 6b). It is important to notice that the maximum concentration is always found here at the highest temperature, as expected (Rittler et al. 2017).

At the first time instant, $t / \tau_{j}=31$, the aggregate diameter shows almost a single bin (Fig. 8a), as expected from the employed monodisperse nucleation model. This 

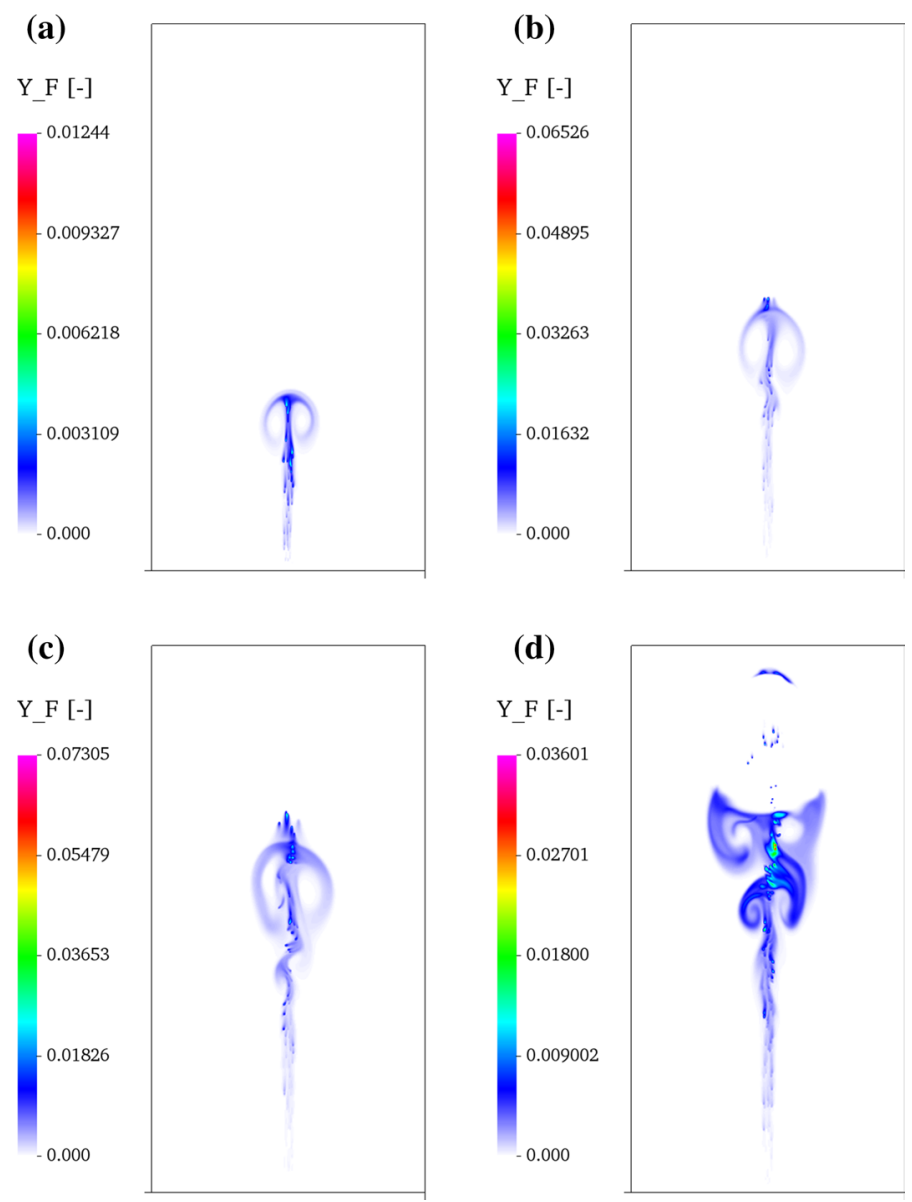

Fig. 4 Spray flame evolution in time represented by a cut plane of the ethanol mass fraction in the gas mixture along the center of the domain. From left to right and from top to down the time is $\mathbf{a} t=31 \tau_{j}, \mathbf{b} 46 \tau_{j}$, $\mathbf{c}$ $61 \tau_{j}, \mathbf{d} 74 \tau_{j}$, respectively. Note that the color scale is changing for each subfigure, following the change in peak mass fraction

indicates that the only dominant process for the number concentration is the nucleation $I$, while coagulation has not started yet (see again Eq. 32). However, the sintering process already starts at this stage, as it can be observed from the primary diameter distribution in Fig. 7a. At $t / \tau_{j}=46$, the primary and aggregate diameters show a wider distribution and the maximum diameter reaches $6 \mathrm{~nm}$ (Figs. 7b, 8b).

\subsubsection{Pre-ignition stage}

At time $t / \tau_{j}=55$ (not shown) the gas mixture temperature starts to increase. Based on classical ignition criteria, corresponding to a temperature increase by $400 \mathrm{~K}$ compared to the initial temperature (Wang and Rutland 2005; Abdelsamie and Thévenin 2017), the ignition starts at $t / \tau_{j}=70.5$. In the interval $55<t / \tau_{j}<70.5$, the evaporation is 

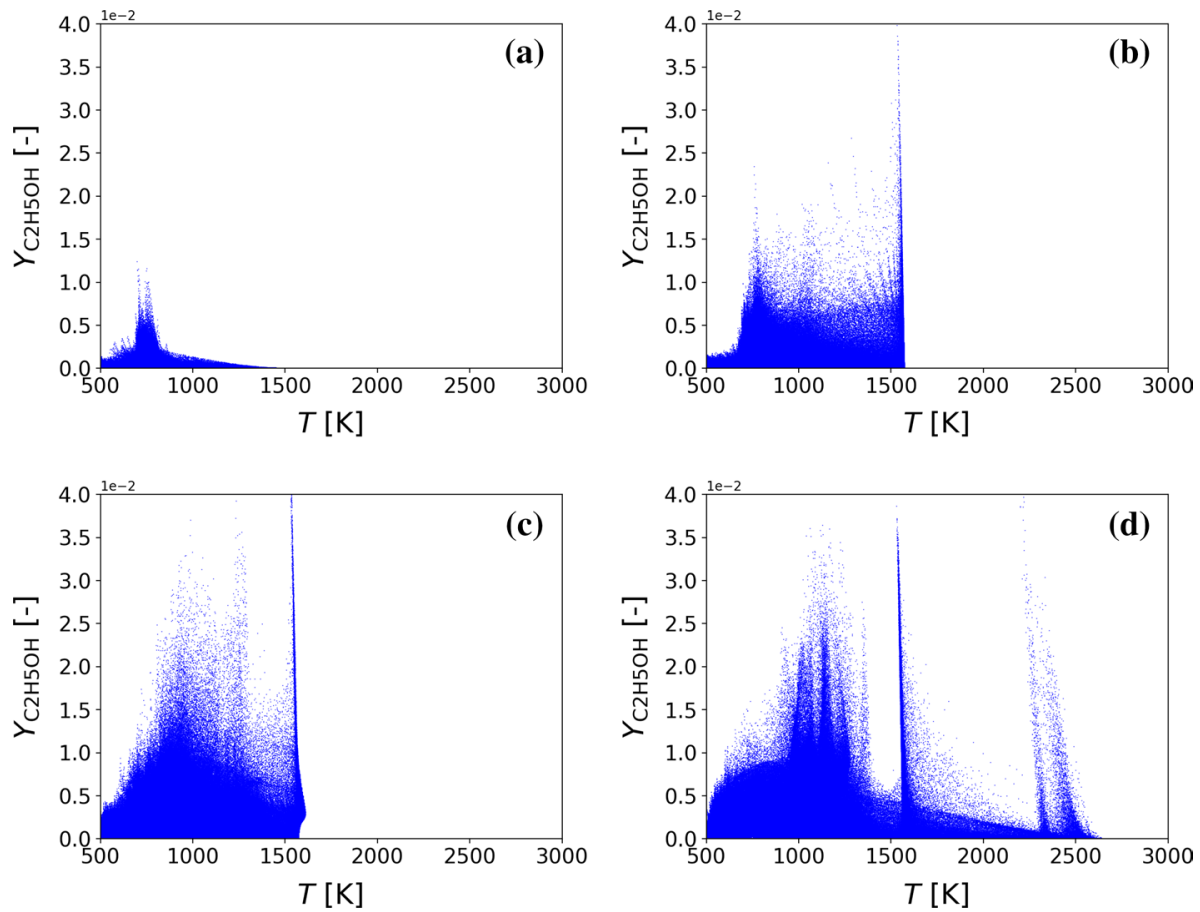

Fig. 5 Scatter plot of ethanol mass fraction in the gas phase versus gas temperature. From left to right and from top to down the time is $\mathbf{a} t=31 \tau_{j}, \mathbf{b} 46 \tau_{j}$, $\mathbf{c} 61 \tau_{j}, \mathbf{d} 74 \tau_{j}$, respectively. Same scales for all subfigures

enhanced and the concentration of ethanol in the gas phase increases as well, as can be seen from Figs. 3c, 4c and 5c.

The nanoparticle number concentration increases accordingly, with a peak found around $1500<T<1600 \mathrm{~K}$; this peak is located at the interface between the ethanol stream and the pilot flame. The primary particles show a distribution similar to a Gaussian one (Fig. 7c). The effect of coagulation is already quite pronounced, and the aggregate diameter shows a more complex distribution (Fig. 8c). The maximum nanoparticle diameter at this stage is about $7 \mathrm{~nm}$.

\subsubsection{Post-ignition stage}

Later on, after ignition occurred (time $t / \tau_{j}>70.5$ ), an unexpected feature is observed: two flames coexist in the simulation, (1) the pilot flame, and (2) a spray flame. This has been observed in the experiment as well (Schneider et al. 2019). The comparison of the flames' structure between DNS and experiment is illustrated in Fig. 9. In the experiment (Fig. 9 Right), the pilot flame covers the circular area just above the burner, whereas the spray flame is represented by the gray color in the middle of the burner. More details about these two flames and their structures have been presented in Ref. Schneider et al. (2019).

Looking back at Figs. $3 \mathrm{~d}$ and $4 \mathrm{~d}$, it is found that the spray droplets are found simultaneously in three different regions: (1) Region with low gas temperature due to evaporation, close to the injector, (2) Region with high mixing and relatively high temperatures, and (3) 

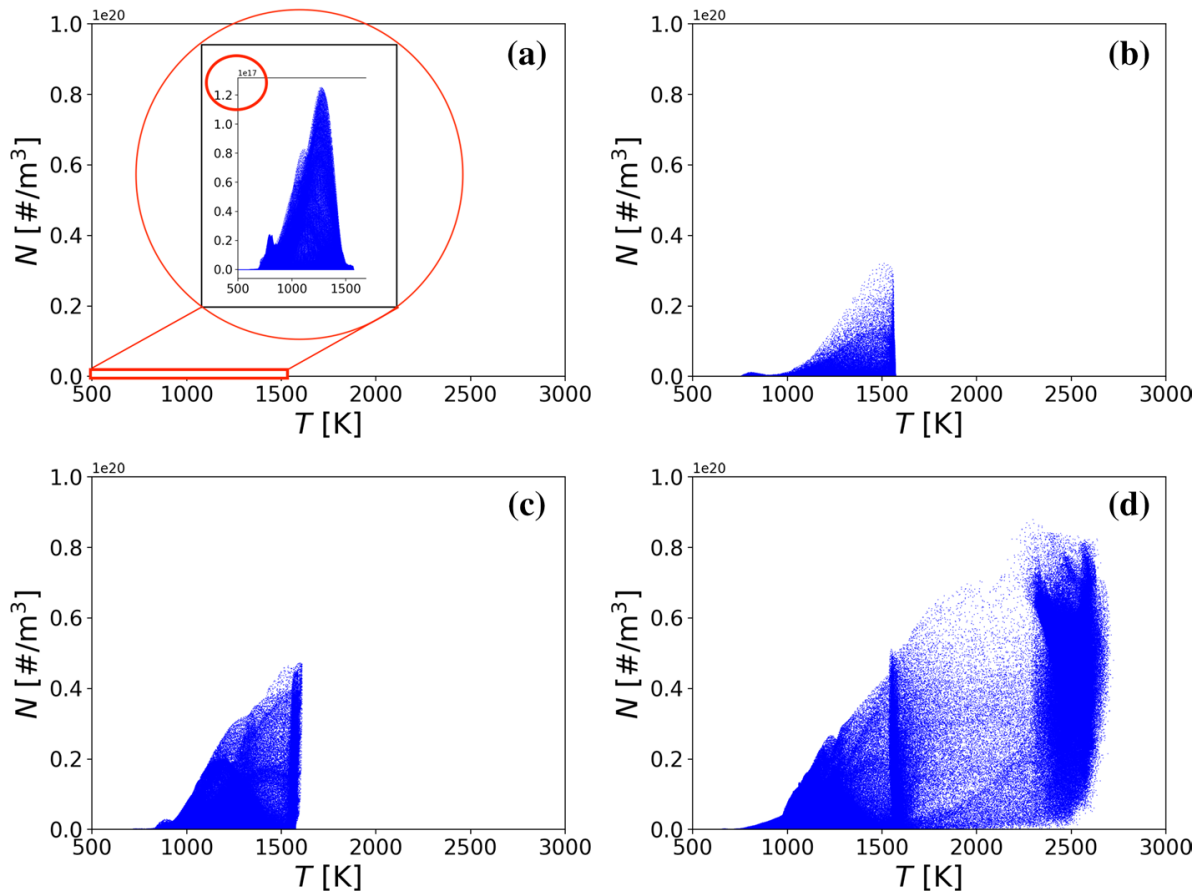

Fig. 6 Scatter plot of nanoparticle number concentration versus gas temperature. From left to right and from top to down the time is $\mathbf{a} t=31 \tau_{j}$, $\mathbf{b} 46 \tau_{j}$, $\mathbf{c} 61 \tau_{j}$, $\mathbf{d} 74 \tau_{j}$, respectively. While the scales are the same for all subfigures, a zoom was necessary in the top-left graph to make the results visible at all

Within the spray flame. This leads to three distinct peaks of gaseous ethanol concentration, as can be observed from Fig. $5 \mathrm{~d}$. The peak of ethanol concentration at $T \approx 2400 \mathrm{~K}$ corresponds to the liquid spray burning within the spray flame.

These three peaks are directly mirrored in the nanoparticle concentration, where three peaks appear as well in Fig. 6d. However, the three peaks are not as clear as previously, the maximum being still found at the highest temperature. As a consequence, the nanoparticle size distributions still reveal a single peak value, as it can be observed from Figs. $7 \mathrm{~d}$ and $8 \mathrm{~d}$. The maximum observed diameter of the nanoparticles is now around $12 \mathrm{~nm}$. The primary particles show a wider range with a nearly Gaussian shape. It is also important to notice that the sintering process now plays a larger role compared to that of coagulation $\left(d_{p}>d_{a}\right)$; this behavior is still under investigation and needs more explanation.

\subsubsection{DNS versus experimental results}

In principle, DNS as a "numerical experiment" is able to take into account all the controlling physicochemical phenomena leading to nanoparticle synthesis in a spray flame. However, a direct quantitative comparison is not possible yet, since (1) experimental measurements are still on-going, and (2) some simplifications have been implemented in the DNS to reduce the computational costs. The main differences between experiment and DNS are (1) different precursors (since a kinetic mechanism for the precursor really used 

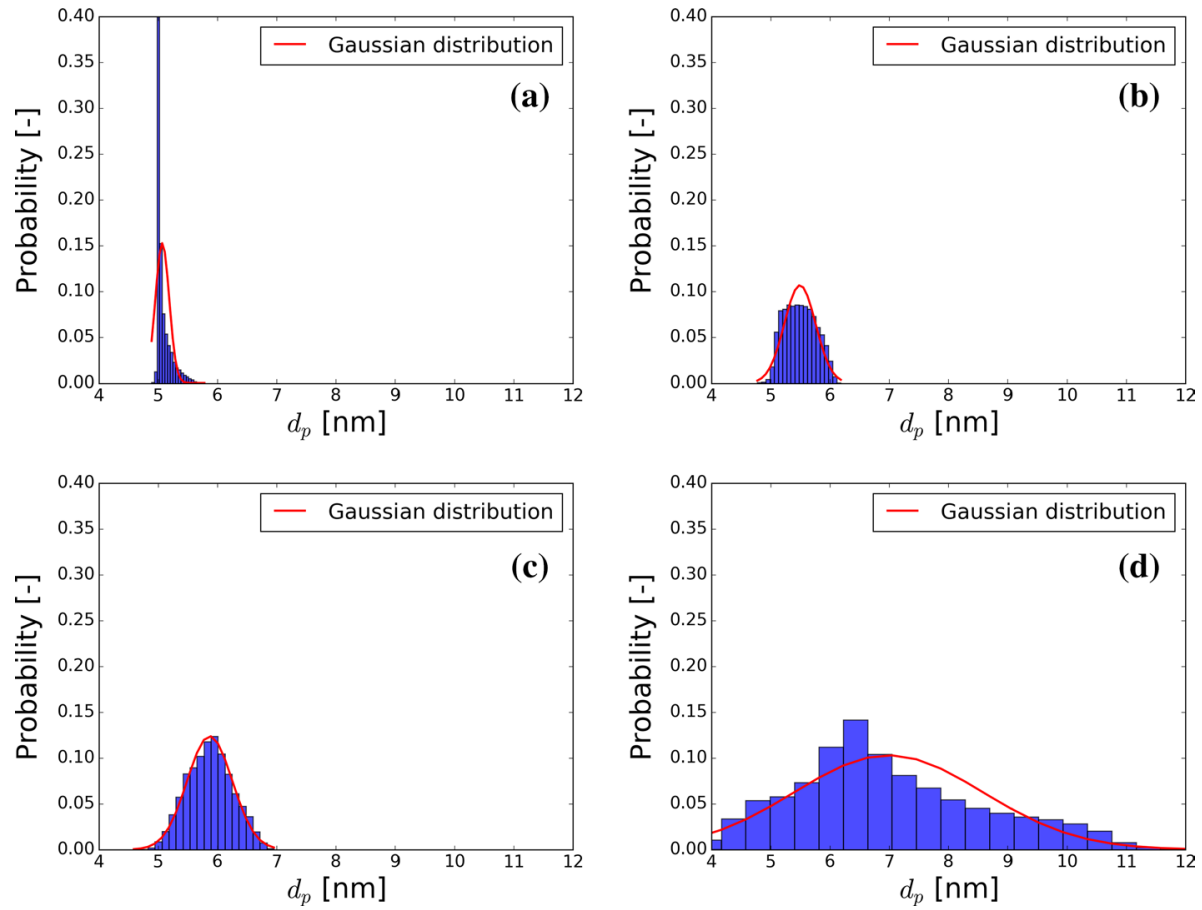

Fig. 7 Normalized particle size distribution (PSD) of the nanoparticle primary diameter. From left to right and from top to down the time is $\mathbf{a} t=31 \tau_{j}$, b $46 \tau_{j}$, c $61 \tau_{j}$, d $74 \tau_{j}$, respectively. Same scales for all subfigures

experimentally is under development at the University of Duisburg-Essen, but not yet available), and (2) a reduced geometrical domain in the DNS. Still, the simplified DNS configuration can already be employed for parameter studies, as described in the next section; this will hopefully be useful to drive further experimental investigations. It is interesting to mention that flame oscillations have been observed for some regimes in the experiments, perturbing the nanoparticle production process. Our simulations and further experiments will hopefully reveal the origin of these oscillations and allow the identification of working solutions to avoid them in the future.

\subsection{Impact of the inlet velocity of the pilot flame}

As it is well know within the research groups producing nanoparticles from sprays, nanoparticles synthesis is highly sensitive with regard to many different parameters (Weise et al. 2015) such as spray droplet size, injection angle, injection speed, solvent, precursor, etc. In this section the impact of the inlet velocity of the pilot flame on the nanoparticle formation is investigated. For that purpose, three different cases are considered here. These cases are similar to the case described in Sect. 3, while changing only the inlet velocity of the pilot flame, $U_{p}$ : (1) Case I: $U_{p}=3.741 \mathrm{~m} / \mathrm{s}$, (2) Case II: $U_{p}=10 \mathrm{~m} / \mathrm{s}$, (3) Case III: $U_{p}=20 \mathrm{~m} / \mathrm{s}$. The results of these three cases are compared at three time instants: $t=46 \tau_{j}, 61 \tau_{j}$, and $74 \tau_{j}$, keeping in mind that the jet time scale $\tau_{j}=3.28 \mu \mathrm{s}$ is the same for all three cases, allowing a direct comparison. 

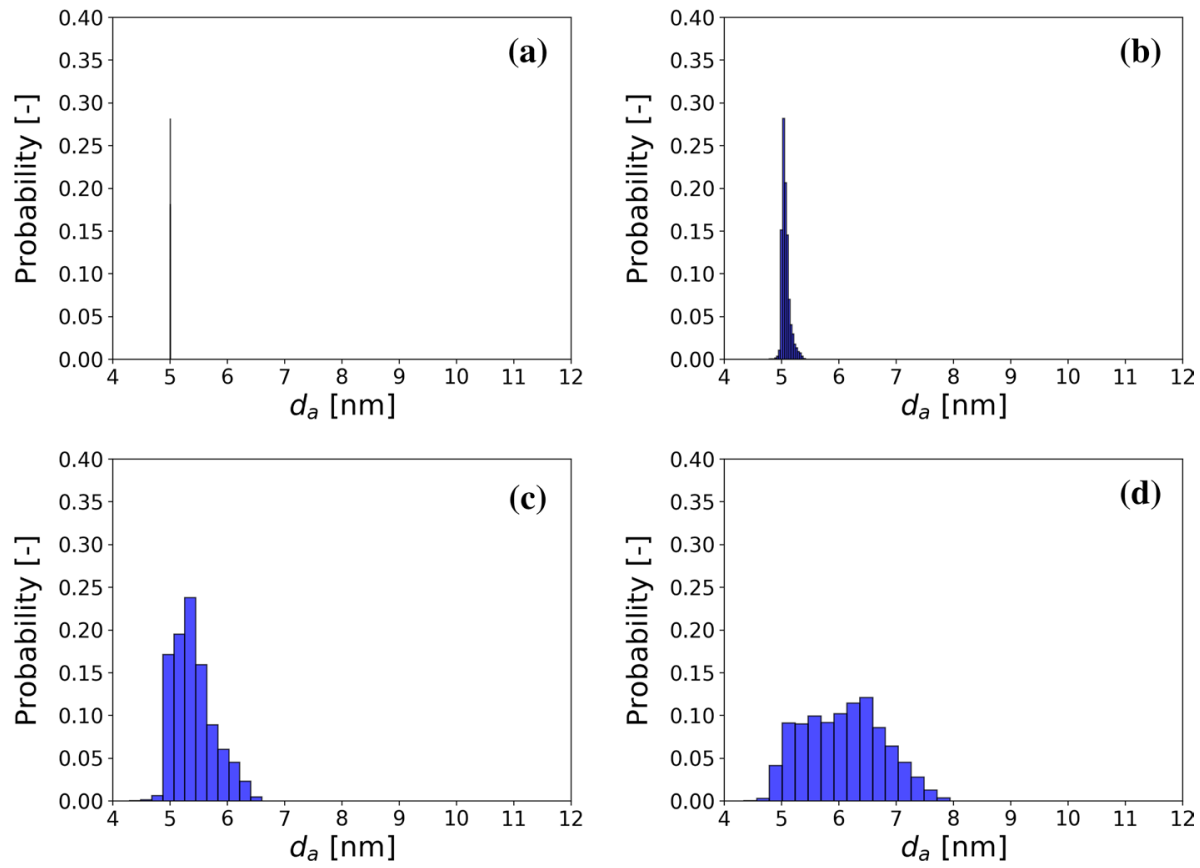

Fig. 8 Normalized particle size distribution of the nanoparticle aggregate diameter. From left to right and from top to down the time is $\mathbf{a} t=31 \tau_{j}, \mathbf{b} 46 \tau_{j}$, $\mathbf{c} 61 \tau_{j}, \mathbf{d} 74 \tau_{j}$, respectively. Same scales for all subfigures

Fig. 9 Schematic diagram showing the similarity between the double flame structure (pilot flame and spray flame) observed in DNS and in the experiments (right figure reprinted from Schneider et al. 2019)

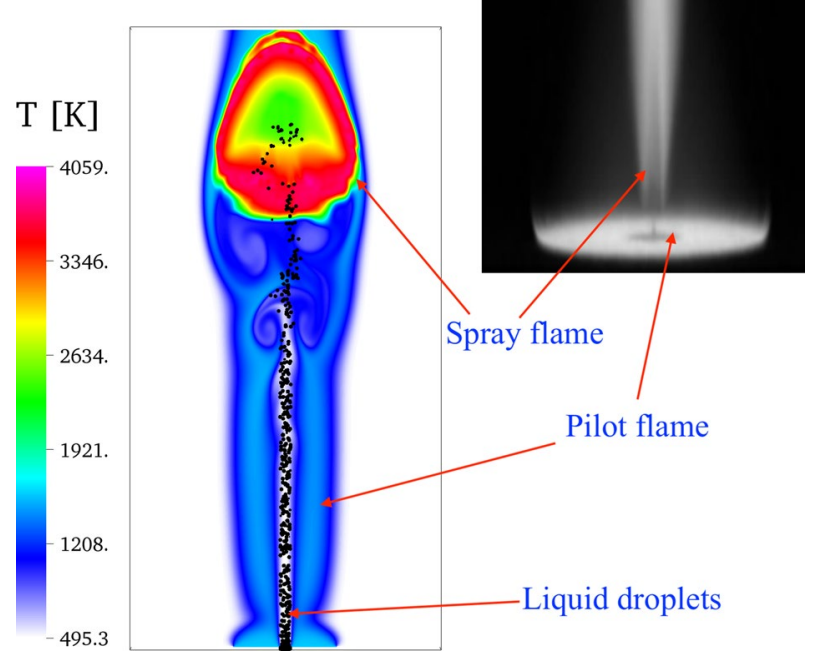



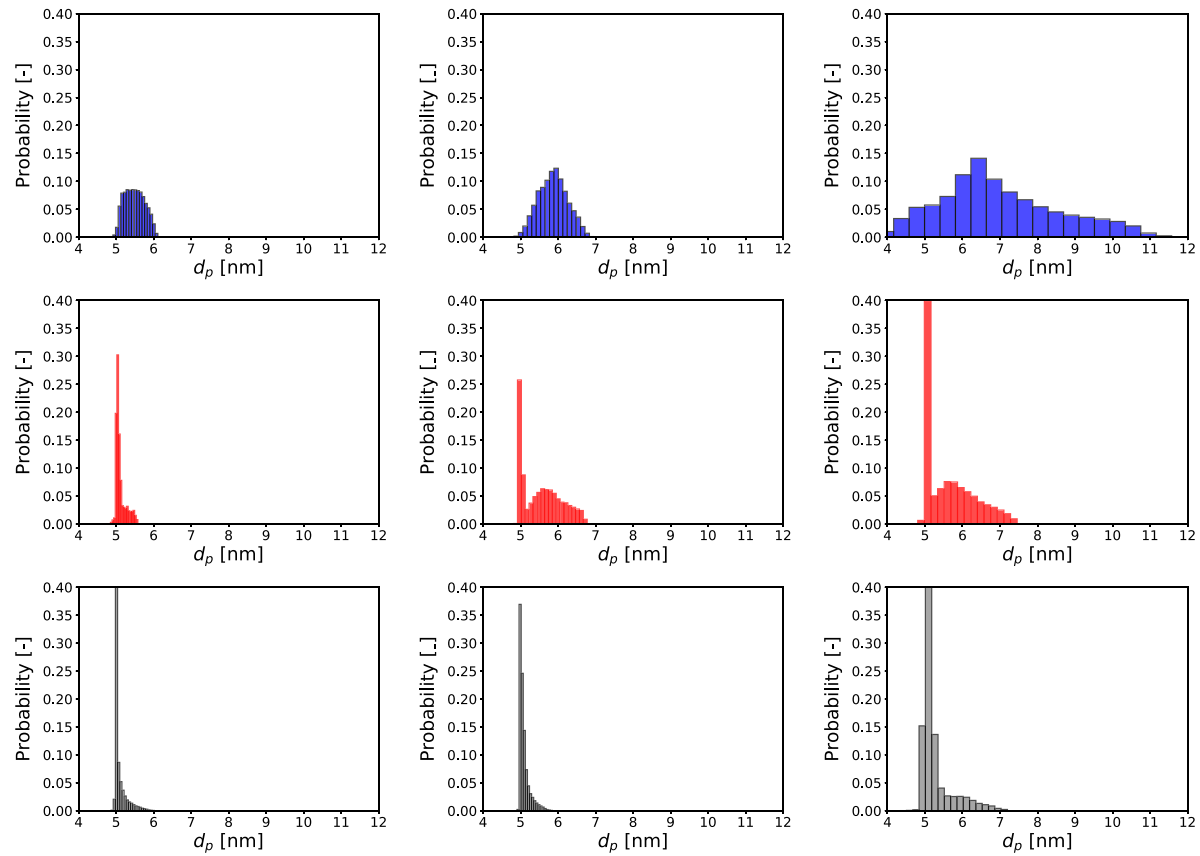

Fig. 10 Normalized particle size distribution for the nanoparticle primary diameter. From top to bottom: Case I, Case II, and Case III, respectively. From left to right the time is $t=46 \tau_{j}, 61 \tau_{j}$, and $74 \tau_{j}$, respectively. Same scales for all subfigures

It is first observed that the ignition delay time of the spray flame $\tau_{g}$ increases with the injection speed of the pilot flame, as follows: (1) Case I: $\tau_{g}=70.5 \tau_{j}$, (2) Case II: $\tau_{g}=79.0 \tau_{j}$, and (3) Case III: $\tau_{g}=86.9 \tau_{j}$. This leads as well to a delay regarding nanoparticle production when increasing the inlet velocity of the pilot flame, as can be seen by looking at Figs. 10 and 11. These figures show the histogram of primary and aggregate nanoparticle diameters, respectively. From Fig. 10, a narrower PSD is found for a larger $U_{p}$, at least at early times. Furthermore, the distribution shows regular behavior, which would facilitate the development of corresponding models.

The same trend is observed for the aggregate diameter in Fig. 11. These results indicate that the inlet velocity of the pilot flame could be used as well as a control parameter to drive the resulting PSD.

\section{Conclusions}

In this work, first direct numerical simulations of a configuration similar to the SpraySynburner have been conducted. The purpose of this burner is to produce nanoparticle materials from a spray flame in a controlled manner. For the presented results, ethanol has been used as solvent and titanium tetraisopropoxide (TTIP) as liquid precursor, with the objective of producing titanium dioxide nanomaterial, $\mathrm{TiO}_{2}$. Liquid spray evaporation and nanoparticle synthesis are represented in the DNS code by three sets of coupled models. The 

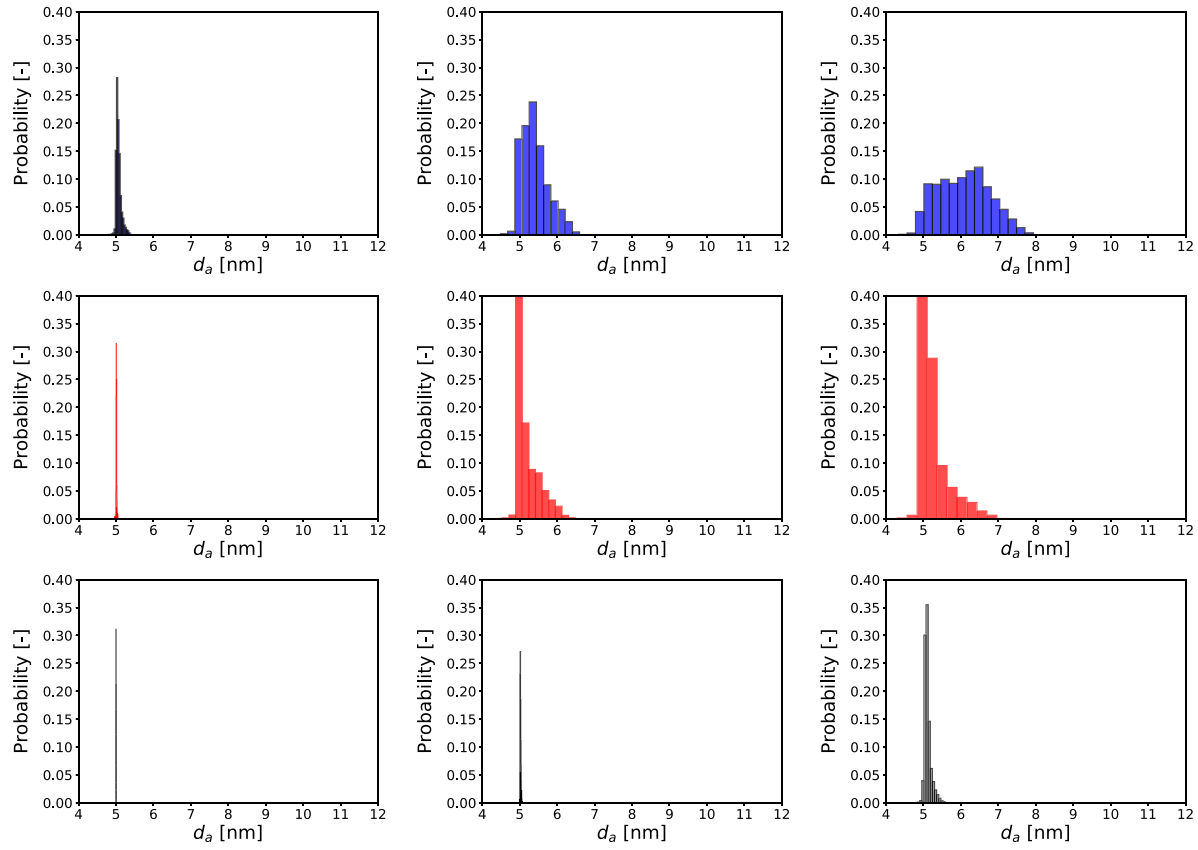

Fig. 11 Normalized particle size distribution for the nanoparticle aggregate diameter. From top to bottom: Case I, Case II, and Case III, respectively. From left to right the time is $t=46 \tau_{j}$, $61 \tau_{j}$, and $74 \tau_{j}$, respectively. Same scales for all subfigures

DNS was able to reproduce the dual-flame behavior observed experimentally, with separate pilot flame and spray flame. The evolution of the particle size distribution in time has been extracted from the DNS and commented physically. It was found that the primary diameter can be modeled using a Gaussian distribution for the conditions considered in this study. However, this is not true for the aggregate diameter. The maximum diameter observed during the DNS simulations was $12 \mathrm{~nm}$.

The impact of the inlet velocity of the pilot flame, $U_{p}$, on the PSD was also investigated. It has been found that increasing $U_{p}$ increases the ignition delay time and reduces the nanoparticle number concentration at a given time; hence, both aggregate and primary nanoparticle diameter decrease. At the same time, a narrower PSD is obtained when increasing $U_{p}$, at least at the beginning of the process. As a consequence, it appears that the pilot flame could be used to control the nanoparticle PSD.

Acknowledgements Open Access funding provided by Projekt DEAL. The financial support of the DFG (Deutsche Forschungsgemeinschaft) within the Project SPP1980 "Nanoparticle Synthesis in Spray Flames SpraySyn: Measurement, Simulation, Processes" is gratefully acknowledged. The computer resources provided by the Gauss Center for Supercomputing/Leibniz Supercomputing Center Munich under Grant pr84qo have been essential to obtain the results presented in this work.

\section{Compliance with ethical standards}

Conflict of interest The authors declare that they have no conflict of interest. 
Open Access This article is licensed under a Creative Commons Attribution 4.0 International License, which permits use, sharing, adaptation, distribution and reproduction in any medium or format, as long as you give appropriate credit to the original author(s) and the source, provide a link to the Creative Commons licence, and indicate if changes were made. The images or other third party material in this article are included in the article's Creative Commons licence, unless indicated otherwise in a credit line to the material. If material is not included in the article's Creative Commons licence and your intended use is not permitted by statutory regulation or exceeds the permitted use, you will need to obtain permission directly from the copyright holder. To view a copy of this licence, visit http://creativecommons.org/licenses/by/4.0/.

\section{References}

Abdelsamie, A., Thévenin, D.: Direct numerical simulation of spray evaporation and autoignition in a temporally-evolving jet. Proc. Combust. Inst. 36(2), 2493 (2017)

Abdelsamie, A., Thévenin, D.: On the behavior of spray combustion in a turbulent spatially-evolving jet investigated by direct numerical simulation. Proc. Combust. Inst. 37(3), 3373 (2019)

Abdelsamie, A., Fru, G., Oster, T., Dietzsch, F., Janiga, G., Thévenin, D.: Towards direct numerical simulations of low-Mach number turbulent reacting and two-phase flows using immersed boundaries. Comput. Fluids 131, 123 (2016)

Abramzon, B., Sirignano, W.A.: Droplet vaporization model for spray combustion calculations. Int. J. Heat Mass Transf. 32(9), 1618 (1989)

Borghesi, G., Mastorakos, E., Cant, R.: Complex chemistry DNS of n-heptane spray autoignition at high pressure and intermediate temperature conditions. Combust. Flame 160, 1254 (2013)

Buesser, B., Gröhn, A., Pratsinis, S.: Sintering rate and mechanism of $\mathrm{TiO}_{2}$ nanoparticles by molecular dynamics. J. Phys. Chem. C 115, 11030-11035 (2011)

Chi, C., Janiga, G., Abdelsamie, A., Zähringer, K., Turányi, T., Thévenin, D.: DNS study of the optimal chemical markers for heat release in syngas flames. Flow Turb. Comb. 98(4), 1117 (2017)

Chi, C., Abdelsamie, A., Thévenin, D.: Direct numerical simulations of hotspot-induced ignition in homogeneous hydrogen-air pre-mixtures and ignition spot tracking. Flow Turb. Comb. 101(1), 103 (2018)

Chi, C., Abdelsamie, A., Thévenin, D.: A directional ghost-cell immersed boundary method for incompressible flows. J. Comput. Phys. 404, 109122 (2020)

Goodwin, D.G., Moffat, H.K., Speth, R.L.: (2015). http://www.cantera.org

Janzen, C., Knipping, J., Rellinghaus, B., Roth, P.: Formation of silica-embedded iron-oxide nanoparticles in low-pressure flames. J. Nanoparticle Res. 5, 589 (2003)

Kammler, H., Mädler, L., Pratsinis, S.: Flame synthesis of nanoparticles. Chem. Eng. Technol. 24(6), $583(2001)$

Kennedy, C., Carpenter, M.: Several new numerical methods for compressible shear layer simulation. Appl. Numer. Math. 14, 397 (1994)

Kitano, T., Nishio, J., Kurose, R., Komori, S.: Effects of ambient pressure, gas temperature and combustion reaction on droplet evaporation. Combust. Flame 161, 551 (2014)

Kruis, F., Kusters, K., Pratsinis, S., Scarlett, B.: A simple model for the evolution of the characteristics of aggregate particles undergoing coagulation and sintering. Aerosol Sci. Technol. 19(4), 514 (1993)

Li, S., Ren, Y., Biswas, P., Tse, S.D.: Flame aerosol synthesis of nanostructured materials and functional devices: processing, modeling, and diagnostics. Prog. Eng. Combust. Sci. 55, 1 (2016)

Mädler, L., Kammler, H., Mueller, R., Pratsinis, S.: Controlled synthesis of nanostructured particles by flame spray pyrolysis. J. Aeros. Sci. 33(2), 369 (2002)

Marinov, N.: A detailed chemical kinetic model for high temperature ethanol oxidation. Int. J. Chem. Kinet. 31(3), 183 (1999)

Mueller, R., Mädler, L., Pratsinis, S.: Nanoparticle synthesis at high production rates by flame spray pyrolysis. Chem. Eng. Sci. 58(10), 1969 (2003)

Panda, S., Pratsinis, S.: Modeling the synthesis of aluminum particles by evaporation-condensation in an aerosol. Nanostruct. Mater. 5, 755 (1995)

Rittler, A., Deng, L., Wlokas, I., Kempf, A.: Large eddy simulations of nanoparticle synthesis from flame spray pyrolysis. Proc. Combust. Inst. 36, 1077 (2017)

Roth, P.: Particle synthesis in flame. Proc. Combust. Inst. 32(2), 1773 (2007) 
Schneider, F., Suleiman, S., Menser, J., Borukhovich, E., Wlokas, I., Kempf, A., Wiggers, H., Schulz, C.: SpraySyn - a standardized burner configuration for nanoparticle synthesis in spray flames. Rev. Sci. Instrum. 90, 085108 (2019)

Wang, Y., Rutland, C.: Effects of temperature and equivalence ratio on the ignition of $n$-heptane fuel spray in turbulent flow. Proc. Combust. Inst. 30, 893 (2005)

Wang, Y., Rutland, C.: Direct numerical simulation of ignition in turbulent $\mathrm{n}$-heptane liquid-fuel spray jets. Combust. Flame 149, 353 (2007)

Weise, C., Menser, J., Kaiser, S., Kempf, A., Wlokas, I.: Numerical investigation of the process steps in a spray flame reactor for nanoparticle synthesis. Proc. Combust. Inst. 35, 2259 (2015) 